\title{
Existence of global solutions with slow decay and unbounded free boundary for a superlinear Stefan problem
}

\author{
MAREK FILA ${ }^{\dagger}$ \\ Institute of Applied Mathematics, Comenius University, Mlynská dolina, 84248 Bratislava, \\ Slovakia \\ AND \\ PHILIPPE SOUPLET ${ }^{*}$ \\ Département de Mathématiques, Université de Picardie, INSSET, 02109 St-Quentin, France
}

[Received 15 June 2000 and in revised form 24 November 2000]

\begin{abstract}
We consider a one-phase Stefan problem for the heat equation with a superlinear reaction term. It is known from a previous work (Ghidouche, Souplet, \& Tarzia [5]) that all global solutions are bounded and decay uniformly to 0 . Moreover, it was shown in Ghidouche, Souplet, \& Tarzia [5] that either: (i) the free boundary converges to a finite limit and the solution decays at an exponential rate, or (ii) the free boundary grows up to infinity and the decay rate is at most polynomial, and it was also proved that small data solutions behave like (i).

Here we prove that there exist global solutions with slow decay and unbounded free boundary, i.e. of type (ii). Also, we establish uniform a priori estimates for all global solutions. Moreover, we provide a correction to an error in the proof of decay from Ghidouche, Souplet, \& Tarzia [5].
\end{abstract}

Keywords: Nonlinear reaction-diffusion equation; free boundary condition; Stefan problem; global existence; decay; a priori estimates

\section{Introduction and results}

We consider the following reaction-diffusion problem with free boundary:

$$
\left\{\begin{array}{l}
u_{t}-u_{x x}=u^{p}, \quad 0<t<T, \quad 0<x<s(t) \\
u(0, x)=u_{0}(x) \geqslant 0, \quad 0<x<s_{0}, \quad s(0)=s_{0}>0 \\
u(t, s(t))=u_{x}(t, 0)=0, \quad 0<t<T \\
s^{\prime}(t)=-u_{x}(t, s(t)), \quad 0<t<T .
\end{array}\right.
$$

Problem (SP) can be viewed as a simple model of a chemically reactive and heat-diffusive liquid surrounded by ice. Here $u \geqslant 0$ represents the temperature of the liquid phase, and the ice is assumed to be at temperature 0 .

Throughout this paper, we suppose $p>1, s_{0}>0$ and $u_{0} \in C^{1}\left(\left[0, s_{0}\right]\right), u_{0} \geqslant 0$, with $\left(u_{0}\right)_{x}(0)=$ $u_{0}\left(s_{0}\right)=0$. It is well known that there exists a unique, maximal in time, classical solution $(u, s)$ of (SP), which satisfies $u \geqslant 0$ and $s^{\prime} \geqslant 0$ (see $[1,4]$ ).

\footnotetext{
†Email: fila@fmph.uniba.sk

*Also at: Laboratoire de Mathématiques Appliquées, UMR CNRS 7641, Université de Versailles, 45 avenue des EtatsUnis, 78035 Versailles, France. Email: souplet@math.uvsq.fr
} 
Global existence, stability and blowup for this problem were studied in [5]. It was proved there that finite time blowup occurs if the initial data $u_{0}$ satisfies a certain energy condition. Concerning global solutions, it was shown that all of them are bounded and decay uniformly to 0 as $t \rightarrow \infty$. Moreover, the following classification of the asymptotic behaviour of global solutions was obtained in [5].

TheOrEm A Let $u$ be a solution of (SP) with $T=\infty$. Then it holds that

$$
\lim _{t \rightarrow \infty}|u(t)|_{\infty}=0
$$

Moreover, if we let $s_{\infty}=\lim _{t \rightarrow \infty} s(t) \leqslant \infty$, then one of the following two possibilities occurs:

(i) (Fast solution) $s_{\infty}<\infty$ and there exist real numbers $C, \alpha>0$ (depending on $u$ ) such that

$$
|u(t)|_{\infty} \leqslant C \mathrm{e}^{-\alpha t}, \quad t \geqslant 0
$$

(ii) (Slow solution) $s_{\infty}=\infty$ and one has the estimates

$$
s(t)=\mathrm{O}\left(t^{2 / 3}\right), \quad t \rightarrow \infty
$$

and

$$
\liminf _{t \rightarrow \infty} s^{2 /(p-1)}(t)|u(t)|_{\infty}>0
$$

hence, in particular,

$$
\liminf _{t \rightarrow \infty} t^{4 /(3(p-1))}|u(t)|_{\infty}>0
$$

Furthermore, it was proved that $u$ is a fast solution whenever the initial datum $u_{0}$ is small in the following sense:

$$
\left|u_{0}\right|_{\infty} \leqslant K \min \left(1, s_{0}^{-2 /(p-1)}\right)
$$

(where $K=K(p)>0$ ). However, the existence of slow solutions was left as an open problem in [5].

The aim of the present paper is to fill this gap by proving the following theorem.

Theorem 1 Let $\phi \in C^{1}\left(\left[0, s_{0}\right]\right)$ satisfy $\phi \geqslant 0, \phi \neq 0$, with $\phi_{x}(0)=\phi\left(s_{0}\right)=0$. Then there exists $\lambda>0$ such that the solution of (SP) with initial data $u_{0}=\lambda \phi$ is a global slow solution (i.e. $u$ satisfies (ii) in Theorem A).

The basic idea of the proof of Theorem 1 is to look for solutions which lie on the boundary of the domain of exponential attraction of 0 . To carry out this idea, we must verify that along each positive ray, this domain is nonempty, open and bounded. Moreover, we must prove that the corresponding limiting solution exists globally. This part of the argument is based on the following uniform a priori estimate for all global solutions of (SP), which may be of independent interest.

Proposition 2 Let $u$ be a solution of (SP) with $T=\infty$. Then there is a constant $C=$ $C\left(\left|u_{0}\right|_{C^{1}}, s_{0}, 1 / s_{0}\right)>0$, such that

$$
\sup _{t \geqslant 0}|u(t)|_{\infty} \leqslant C
$$

where $C$ remains bounded for $\left|u_{0}\right|_{C^{1}}, s_{0}$ and $1 / s_{0}$ bounded. 
The proof of this estimate relies on scaling and energy arguments similar to those in [6]. However, some additional difficulties arise due to the free-boundary condition (and moving domain). These arguments are also related to those used in [5] in the proof of decay of global solutions. However, it appeared that the proof of (1.1) in [5] was not completely correct in the case $s_{\infty}=\infty$, because some terms related to the free boundary had been overlooked. We will here fill this gap and give a correct proof of the decay property (1.1) and of the a priori estimates at the same time. Moreover, this proof is simpler because one no longer needs to treat the cases $s_{\infty}<\infty$ and $s_{\infty}=\infty$ separately.

Let us mention that the uniqueness of $\lambda$ in Theorem 1, as well as the precise decay/growth rate of $|u(t)|_{\infty}$ and $s(t)$, remain open questions.

Earlier results on blowup for problem (SP) can be found in [1,7], where the shape of some blowup solutions was studied. On the other hand, the existence of global solutions to (SP) was studied in [2,3]. It was proved in [2] that small data yield global fast solutions, but the result was obtained only for $p>2$, while the corresponding result of [5: Theorem 3.2] holds for all $p>1$. In [3], it was shown for $p>2$ that global fast solutions are stable. In a forthcoming publication [8], it will be shown that the stability property remains actually true for all $p>1$.

\section{Proofs}

Let us first recall the basic energy identity

$$
E\left(u\left(t_{2}\right)\right)-E\left(u\left(t_{1}\right)\right)=-\int_{t_{1}}^{t_{2}} \int_{0}^{s(t)} u_{t}^{2}(t, x) \mathrm{d} x \mathrm{~d} t-\frac{1}{2} \int_{t_{1}}^{t_{2}} s^{\prime 3}(t) \mathrm{d} t, \quad 0 \leqslant t_{1} \leqslant t_{2}<T,
$$

where

$$
E(u(t)):=\int_{0}^{s(t)}\left(\frac{\left(u_{x}\right)^{2}}{2}-\frac{u^{p+1}}{p+1}\right)(t, x) \mathrm{d} x .
$$

Also, as a consequence of the blowup result of [5: Theorem 2.1], we know that $T=\infty$ implies $E(u(t)) \geqslant 0$ for all $t \geqslant 0$, hence

$$
\int_{0}^{\infty} \int_{0}^{s(t)} u_{t}^{2}(t, x) \mathrm{d} x \mathrm{~d} t+\frac{1}{2} \int_{0}^{\infty} s^{\prime 3}(t) \mathrm{d} t \leqslant E(u(0))<\infty .
$$

We will first prove Theorem 1 assuming Proposition 2.

Proof of Theorem 1. To emphasize the dependence of $u$ on the initial data when necessary, we will denote the solution of (SP) by $u\left(u_{0} ; \cdot\right)$ (and $s(t), s_{\infty}$ and the maximal existence time $T$ accordingly).

We define

$$
\Sigma=\left\{\lambda>0 ; T(\lambda \phi)=\infty \text { and } s_{\infty}(\lambda \phi)<\infty\right\} .
$$

First, we have $\Sigma \neq \emptyset$ (it contains $(0, \varepsilon)$ for $\varepsilon>0$ small—see the paragraph after Theorem A). On the other hand, for $\lambda$ sufficiently large, we have $E(\lambda \phi)<0$ hence $T(\lambda \phi)<\infty$ (see before formula (2.1)). Therefore $\Sigma$ is bounded.

Let $\lambda^{*}=\sup \Sigma \in(0, \infty), v=u\left(\lambda^{*} \phi ; \cdot\right), \sigma=s\left(\lambda^{*} \phi ; \cdot\right)$ and $\tau=T\left(\lambda^{*} \phi\right)$.

We first claim that $\tau=\infty$. Indeed, by continuous dependence (see [4, 8]), for each fixed $t \in$ $[0, \tau), u(\lambda \phi ; t)$ approaches $v(t)$ in $L^{\infty}(0, \infty)$ and $s(\lambda \phi ; t) \rightarrow \sigma(t)$, as $\lambda \uparrow \lambda^{*}$ (one here extends $u(t)$ by 0 on $(s(t), \infty)$ ). Since $T(\lambda \phi)=\infty$ for all $\lambda \in\left(0, \lambda^{*}\right)$, it follows from Proposition 2 
that $|v(t)|_{\infty} \leqslant C$ for all $t \in[0, \tau)$. Therefore, $\tau=\infty$ since nonglobal solutions must satisfy $\lim \sup _{t \rightarrow T}|u(t)|_{\infty}=\infty$ (see [1: Proposition 3.1]).

Next we claim that $\sigma_{\infty}=\infty$. Assume $\sigma_{\infty}<\infty$ for contradiction. Since $|v(t)|_{\infty} \rightarrow 0$ as $t \rightarrow \infty$ by Theorem A, we will have

$$
\left|v\left(t_{0}\right)\right|_{\infty}<K \min \left(1, \sigma\left(t_{0}\right)^{-2 /(p-1)}\right)
$$

for some large $t_{0}$. By continuous dependence, it will follow that

$$
\left|u\left(\lambda \phi ; t_{0}\right)\right|_{\infty}<K \min \left(1, s\left(\lambda \phi ; t_{0}\right)^{-2 /(p-1)}\right)
$$

for $\lambda>\lambda^{*}$ sufficiently close to $\lambda^{*}$. But this implies that $s_{\infty}(\lambda \phi)<\infty$ (see the paragraph after Theorem A), which is a contradiction with the definition of $\lambda^{*}$.

The proof of Theorem 1 is complete.

Proof of (1.1) in Theorem A. Assume for contradiction that $\ell=\lim \sup _{t \rightarrow \infty}|u(t)|_{\infty}>0$.

If $\ell=\infty$, then there is a sequence $t_{n} \rightarrow \infty$ such that $\left|u\left(t_{n}\right)\right|_{\infty}=\sup _{\left[0, t_{n}\right]}|u(t)|_{\infty} \rightarrow \infty$, as $n \rightarrow \infty$. If $\ell<\infty$, let $t_{0}>0$ be such that $\sup _{\left[t_{0}, \infty\right)}|u(t)|_{\infty} \leqslant 3 \ell / 2$. Then there is a sequence $t_{n} \rightarrow \infty$ such that $\left|u\left(t_{n}\right)\right|_{\infty} \geqslant 3 \ell / 4 \geqslant \frac{1}{2} \sup _{\left[t_{0}, t_{n}\right]}|u(t)|_{\infty}$. In both cases, we thus have a sequence $t_{n} \rightarrow \infty$ and $\varepsilon>0$ such that $\sigma_{n}:=\left|u\left(t_{n}\right)\right|_{\infty} \geqslant \frac{1}{2} \sup _{\left[t_{0}, t_{n}\right]}|u(t)|_{\infty} \geqslant \varepsilon$. Pick $x_{n} \in\left[0, s\left(t_{n}\right)\right)$ such that $u\left(t_{n}, x_{n}\right)=\sigma_{n}$ and set

$$
\lambda_{n}=\sigma_{n}^{-(p-1) / 2} \leqslant \varepsilon^{-(p-1) / 2} .
$$

We extend $u(t, \cdot)$ by 0 on $(s(t), \infty)$ and define the rescaled function

$$
v_{n}(\tau, y)=\lambda_{n}^{2 /(p-1)} u\left(t_{n}+\lambda_{n}^{2} \tau, x_{n}+\lambda_{n} y\right)
$$

for $(\tau, y) \in \widetilde{D}_{n}=\left\{(\tau, y) ; \lambda_{n}^{-2}\left(t_{0}-t_{n}\right) \leqslant \tau \leqslant 0\right.$ and $\left.-\lambda_{n}^{-1} x_{n} \leqslant y<\infty\right\}$. Also we denote

$$
y_{1}=-\lambda_{n}^{-1} x_{n}, \quad y_{2}(\tau)=\lambda_{n}^{-1}\left(s\left(t_{n}+\lambda_{n}^{2} \tau\right)-x_{n}\right)
$$

and

$$
D_{n}=\left\{(\tau, y) ; \lambda_{n}^{-2}\left(t_{0}-t_{n}\right) \leqslant \tau \leqslant 0 \text { and } y_{1} \leqslant y<y_{2}(\tau)\right\},
$$

which corresponds to $x<s(t)$. The function $v_{n}$ satisfies $v_{n}(0,0)=1,0 \leqslant v_{n} \leqslant 2$ in $\widetilde{D}_{n}$, and

$$
\partial_{\tau} v_{n}-\partial_{y}^{2} v_{n}=v_{n}^{p} \quad \text { in } D_{n} .
$$

Now we want to get local estimates of derivatives of $v_{n}$ in compact subsets of the form $Q_{m}=$ $[-m, 0] \times[0, m]$ for $m \geqslant 1$. Observe that $Q_{m}$ is not necessarily included in $D_{n}$ for $n$ large (because we might have $\left.s\left(t_{n}-m \lambda_{n}^{2}\right)<x_{n}\right)$. So we cannot directly apply parabolic regularity theory to equation (2.3) to derive these estimates. Also, if we want to extend equation (2.3) to the larger domain $\widetilde{D}_{n} \supset Q_{m}$, we will inherit extra Dirac masses, corresponding to the discontinuity of $\partial_{y} v_{n}$ at the free boundary. (These are the points which had been overlooked in the proof of [5].) To overcome this, we will derive local $L^{2}$ estimates of derivatives by employing the local-energy method in $D_{n}$ and controlling the contributions from the free boundary. 
LEMMA 2.1 For all $m \geqslant 1$ and $n \geqslant N(m)$ large, it holds that

$$
\int_{0}^{m} v_{n, y}^{2}(\tau, y) \mathrm{d} y \leqslant C(m), \quad-m \leqslant \tau \leqslant 0
$$

and

$$
\int_{-m}^{0} \int_{0}^{m}\left(v_{n, \tau}^{2}+v_{n, y}^{2}\right)(\tau, y) \mathrm{d} y \mathrm{~d} \tau \leqslant C(m)
$$

Proof of Lemma 2.1 Let $\varphi \in C_{0}^{\infty}(\mathbb{R})$ be such that $\varphi=1$ on $[0, m]$ and set $v=v_{n}$. For $n$ large enough and $-m \leqslant \tau \leqslant 0$, using $v_{y}\left(\tau, y_{1}(\tau)\right)=v\left(\tau, y_{2}(\tau)\right)=0$, we have

$$
\begin{aligned}
\frac{1}{2} \frac{\mathrm{d}}{\mathrm{d} \tau} \int_{y_{1}}^{y_{2}(\tau)} v^{2}(\tau, y) \varphi^{2}(y) \mathrm{d} y & =\int_{y_{1}}^{y_{2}} v v_{\tau} \varphi^{2}=\int_{y_{1}}^{y_{2}} v^{p+1} \varphi^{2}+\int_{y_{1}}^{y_{2}} v_{y y} v \varphi^{2} \\
& =\int_{y_{1}}^{y_{2}} v^{p+1} \varphi^{2}+\left[v_{y} v \varphi^{2}\right]_{y_{1}}^{y_{2}}-\int_{y_{1}}^{y_{2}} v_{y}^{2} \varphi^{2}-2 \int_{y_{1}}^{y_{2}} v v_{y} \varphi \varphi_{y} \\
& \leqslant \int_{y_{1}}^{y_{2}} v^{p+1} \varphi^{2}-\frac{1}{2} \int_{y_{1}}^{y_{2}} v_{y}^{2} \varphi^{2}+C \int_{y_{1}}^{y_{2}} v^{2} \varphi_{y}^{2} .
\end{aligned}
$$

Therefore, since $0 \leqslant v \leqslant 2$, we have

$$
\begin{aligned}
\int_{-m}^{0} \int_{y_{1}}^{y_{2}(\tau)} v_{y}^{2}(\tau, y) \varphi^{2}(y) \mathrm{d} y & \\
& \leqslant C \int_{-m}^{0} \int_{y_{1}}^{y_{2}}\left(v^{p+1} \varphi^{2}+v^{2} \varphi_{y}^{2}\right)+\int_{y_{1}}^{y_{2}(-m)} v^{2}(-m, y) \varphi^{2} \leqslant C(m) .
\end{aligned}
$$

Next we put $E_{\varphi}(\tau)=\int_{y_{1}}^{y_{2}(\tau)}\left(\frac{v_{y}^{2}}{2}-\frac{v^{p+1}}{p+1}\right)(\tau, y) \varphi^{2}(y) \mathrm{d} y$, and we compute

$$
\begin{aligned}
\frac{\mathrm{d} E_{\varphi}(\tau)}{\mathrm{d} \tau}= & \int_{y_{1}}^{y_{2}}\left(v_{y \tau} v_{y}-v^{p} v_{\tau}\right) \varphi^{2}+\frac{\mathrm{d} y_{2}}{\mathrm{~d} \tau} \frac{v_{y}^{2}\left(\tau, y_{2}(\tau)\right)}{2} \varphi^{2}\left(y_{2}(\tau)\right) \\
= & \left(v_{\tau} v_{y} \varphi^{2}\right)\left(\tau, y_{2}(\tau)\right)-\int_{y_{1}}^{y_{2}} v_{\tau} v_{y y} \varphi^{2}-2 \int_{y_{1}}^{y_{2}} v_{\tau} v_{y} \varphi \varphi_{y} \\
& -\int_{y_{1}}^{y_{2}} v^{p} v_{\tau} \varphi^{2}+\frac{\mathrm{d} y_{2}}{\mathrm{~d} \tau} \frac{v_{y}^{2}\left(\tau, y_{2}(\tau)\right)}{2} \varphi^{2}\left(y_{2}(\tau)\right) .
\end{aligned}
$$

On the other hand, we have $0=\frac{\mathrm{d}}{\mathrm{d} \tau}\left[v\left(\tau, y_{2}(\tau)\right)\right]=\left(v_{\tau}+\frac{\mathrm{d} y_{2}}{\mathrm{~d} \tau} v_{y}\right)\left(\tau, y_{2}(\tau)\right)$, hence

$$
\left\{v_{\tau} v_{y} \varphi^{2}+\frac{\mathrm{d} y_{2}}{\mathrm{~d} \tau} \frac{v_{y}^{2}}{2} \varphi^{2}\right\}\left(\tau, y_{2}(\tau)\right)=-\left(\frac{\mathrm{d} y_{2}}{\mathrm{~d} \tau} \frac{v_{y}^{2}}{2} \varphi^{2}\right)\left(\tau, y_{2}(\tau)\right) \leqslant 0 .
$$

It follows that

$$
\frac{\mathrm{d} E_{\varphi}(\tau)}{\mathrm{d} \tau} \leqslant-\int_{y_{1}}^{y_{2}} v_{\tau}^{2} \varphi^{2}-2 \int_{y_{1}}^{y_{2}} v_{\tau} v_{y} \varphi \varphi_{y} \leqslant-\frac{1}{2} \int_{y_{1}}^{y_{2}} v_{\tau}^{2} \varphi^{2}+C \int_{y_{1}}^{y_{2}} v_{y}^{2} \varphi_{y}^{2} .
$$


For $-m \leqslant \alpha \leqslant \beta \leqslant 0$, by integrating in $\tau$ over $[\alpha, \beta]$, we get

$$
\int_{\alpha}^{\beta} \int_{y_{1}}^{y_{2}} v_{\tau}^{2} \varphi^{2}+\int_{y_{1}}^{y_{2}(\beta)} v_{y}^{2}(\beta) \varphi^{2} \leqslant \int_{y_{1}}^{y_{2}(\alpha)} v_{y}^{2}(\alpha) \varphi^{2}+C(m),
$$

where (2.4) and $0 \leqslant v \leqslant 2$ have been used. A further integration in $\alpha$ over $[\beta-1, \beta]$ yields in particular

$$
\int_{y_{1}}^{y_{2}(\beta)} v_{y}^{2}(\beta) \varphi_{y}^{2} \leqslant \int_{\beta-1}^{\beta} \int_{y_{1}}^{y_{2}(\alpha)} v_{y}^{2}(\alpha) \varphi_{y}^{2}+C(m) \leqslant C^{\prime}(m)
$$

(where (2.4) and (2.5) have been used with $m+1$ instead of $m$ ), hence

$$
\int_{0}^{m} v_{y}^{2}(\tau) \leqslant C(m), \quad-m \leqslant \tau \leqslant 0 .
$$

Finally, returning to (2.5) with $\alpha=-m, \beta=0$, we get

$$
\int_{-m}^{0} \int_{0}^{m} v_{\tau}^{2} \leqslant C(m)
$$

and the lemma follows from (2.6) and (2.7).

From Lemma 2.1 and $0 \leqslant v_{n} \leqslant 2$, by the compact embeddings $H^{1}\left(Q_{m}\right) \subset L^{p}\left(Q_{m}\right)$ and $H^{1}([0, m]) \subset C([0, m])$, it follows that (some subsequence of) $v_{n}$ converges in $L_{\text {loc }}^{p}((-\infty, 0] \times$ $[0, \infty))$ to some function $w \in L_{\mathrm{loc}}^{p}((-\infty, 0] \times[0, \infty))$ and that $v_{n}(0, \cdot)$ converges in $C_{\mathrm{loc}}([0, \infty))$ to some function $z \in C([0, \infty))$ which satisfies $z(0)=1$.

Next, we will use the following two lemmas.

Lemma 2.2 Let $Q=(-\infty, 0) \times(0, \infty)$. Then it holds that

$$
w_{\tau}-w_{y y}=w^{p} \quad \text { in } \mathcal{D}^{\prime}(Q) .
$$

Proof of Lemma 2.2 Take $\varphi \in C_{0}^{\infty}(Q)$, with $\operatorname{supp}(\varphi) \subset[-m, 0) \times(0, m]$. For each $\tau \in[-m, 0]$ and $m$ large enough, we have

$$
\begin{aligned}
\int_{0}^{\infty}\left(\left(v_{n, \tau}-v_{n}^{p}\right) \varphi\right)(\tau, y) \mathrm{d} y & =\int_{0}^{y_{2}(\tau)} v_{n, y y}(\tau) \varphi=\left(v_{n, y} \varphi\right)\left(\tau, y_{2}(\tau)\right)-\int_{0}^{y_{2}(\tau)} v_{n, y}(\tau) \varphi_{y} \\
& =-\lambda_{n}^{\frac{p+1}{p-1}} s^{\prime}\left(t_{n}+\lambda_{n} \tau\right) \varphi\left(y_{2}(\tau)\right)+\int_{0}^{y_{2}(\tau)} v_{n}(\tau) \varphi_{y y}
\end{aligned}
$$

(since $\left.v_{n}\left(\tau, y_{2}(\tau)\right)=0\right)$. Therefore,

$$
\begin{aligned}
\left|\left\langle v_{n, \tau}-v_{n, y y}-v_{n}^{p}, \varphi\right\rangle_{\mathcal{D}^{\prime}, \mathcal{D}}\right| & =\left|\int_{-m}^{0} \int_{0}^{\infty}\left(\left(v_{n, \tau}-v_{n}^{p}\right) \varphi-v_{n} \varphi_{y y}\right)(\tau, y) \mathrm{d} y \mathrm{~d} \tau\right| \\
& =\lambda_{n}^{\frac{p+1}{p-1}}\left|\int_{-m}^{0} s^{\prime}\left(t_{n}+\lambda_{n} \tau\right) \varphi\left(y_{2}(\tau)\right) \mathrm{d} \tau\right| \\
& \leqslant \lambda_{n}^{\frac{p+1}{p-1}-1}|\varphi|_{\infty} \int_{t_{n}-m \lambda_{n}}^{t_{n}} s^{\prime}(t) \mathrm{d} t \\
& \leqslant \lambda_{n}^{\frac{2}{p-1}}|\varphi|_{\infty}\left(\int_{t_{n}-m \lambda_{n}}^{t_{n}} s^{\prime 3}\right)^{1 / 3}\left(m \lambda_{n}\right)^{2 / 3} \rightarrow 0, \quad \text { as } n \rightarrow \infty
\end{aligned}
$$

since $\lambda_{n}$ is bounded, $t_{n} \rightarrow \infty$ and $\int_{0}^{\infty} s^{\prime 3}(t) \mathrm{d} t<\infty$ (see (2.1)). The lemma follows. 
Lemma 2.3 It holds that $w_{\tau}=0$ in $\mathcal{D}^{\prime}(Q)$.

Proof of Lemma 2.3 We have

$$
\begin{aligned}
\int_{-m}^{0} \int_{0}^{m} v_{n, \tau}^{2} & =\lambda_{n}^{\frac{4 p}{p-1}} \int_{-m}^{0} \int_{0}^{m} u_{t}^{2}\left(t_{n}+\lambda_{n}^{2} \tau, x_{n}+\lambda_{n} y\right) \mathrm{d} y \mathrm{~d} \tau \\
& \leqslant \lambda_{n}^{\frac{4 p}{p-1}-3} \int_{t_{n}-m \lambda_{n}}^{t_{n}} \int_{0}^{s(t)} u_{t}^{2}(t, x) \mathrm{d} x \mathrm{~d} t \rightarrow 0, \quad \text { as } n \rightarrow \infty
\end{aligned}
$$

since $\lambda_{n}$ is bounded, $t_{n} \rightarrow \infty, \frac{4 p}{p-1}>3$ and $\int_{0}^{\infty} \int_{0}^{s(t)} u_{t}^{2}(t, x) \mathrm{d} x \mathrm{~d} t<\infty$ (see (2.1)). The lemma follows.

End of proof of (1.1). By Lemmas 2.2 and 2.3 we have obtained a function $w \geqslant 0$, bounded and continuous on $[0, \infty)$, such that $-w_{y y}=w^{p}$, hence $w$ is concave. Therefore $w \equiv 0$ : a contradiction with $w(0)=z(0)=1$.

Proof of Proposition 2. First from the local theory for problem (SP) (see [4]), for each $M>1$ there exists $\delta>0$ such that, if $\left|u_{0}\right|_{C^{1}}<M$ and $1 / M<s_{0}<M$, then $T>\delta$ and $|u(t)|_{\infty}<2 M$ on $[0, \delta]$.

Assume that the result is false. Then there exist $M>0$ and a sequence of global solutions $\left(u_{n}, s_{n}\right)$ of (SP), such that

$$
1 / M<s_{n}(0)<M, \quad\left|u_{n}(0)\right|_{C^{1}}<M \quad \text { and } \quad \sup _{t \geqslant 0}\left|u_{n}(t)\right|_{\infty} \rightarrow \infty, \quad \text { as } n \rightarrow \infty .
$$

For all large $n$ there exist $t_{n} \geqslant \delta$ and $x_{n} \in\left[0, s_{n}\left(t_{n}\right)\right)$ such that

$$
\sup _{t \geqslant 0}\left|u_{n}(t)\right|_{\infty}=u_{n}\left(t_{n}, x_{n}\right)=: \sigma_{n} \text {. }
$$

We define $\lambda_{n}=\sigma_{n}^{-(p-1) / 2} \rightarrow 0$, as $n \rightarrow \infty$, we extend $u_{n}(t, \cdot)$ by 0 on $\left(s_{n}(t), \infty\right)$, and we rescale $u_{n}$ as in (2.2), with $t_{0}=0$ and $u_{n}$ instead of $u$. Observe that $\lambda_{n}^{-2} t_{n} \rightarrow \infty$ as $n \rightarrow \infty$. The function $v_{n}$ now satisfies $(2.3)$ and $v_{n}(0,0)=1, v_{n} \leqslant 1$ in $\widetilde{D}_{n}$.

Therefore, Lemma 2.1 applies and yields a subsequence of $v_{n}$, which converges in the same way as before. Next, we claim that the conclusions of Lemmas 2.2 and 2.3 are still true. Indeed, in view of (2.1), the calculation in the proof of Lemma 2.2 yields

$$
\begin{aligned}
\left|\left\langle v_{n, \tau}-v_{n, y y}-v_{n}^{p}, \varphi\right\rangle_{\mathcal{D}^{\prime}, \mathcal{D}}\right| & \leqslant \lambda_{n}^{\frac{2}{p-1}}|\varphi|_{\infty}\left(\int_{0}^{\infty} s_{n}^{\prime 3}\right)^{1 / 3}\left(m \lambda_{n}\right)^{2 / 3} \\
& \leqslant \lambda_{n}^{\frac{2}{p-1}+\frac{2}{3}} m^{2 / 3}|\varphi|_{\infty}\left(2 E\left(u_{n}(0)\right)\right)^{1 / 3} \\
& \leqslant \lambda_{n}^{\frac{2}{p-1}+\frac{2}{3}} m^{2 / 3}|\varphi|_{\infty} C(M),
\end{aligned}
$$

and the right-hand side goes to 0 as $n \rightarrow \infty$. Similarly, in view of (2.1), the calculation in the proof of Lemma 2.3 yields

$$
\begin{aligned}
\int_{-m}^{0} \int_{0}^{m} v_{n, \tau}^{2} & \leqslant \lambda_{n}^{\frac{4 p}{p-1}-3} \int_{0}^{\infty} \int_{0}^{s_{n}(t)} u_{n, t}^{2}(t, x) \mathrm{d} x \mathrm{~d} t \\
& \leqslant \lambda_{n}^{\frac{4 p}{p-1}-3} E\left(u_{n}(0)\right) \leqslant \lambda_{n}^{\frac{4 p}{p-1}-3} C(M) \rightarrow 0, \quad \text { as } n \rightarrow \infty .
\end{aligned}
$$

We then obtain a contradiction exactly as in the end of the proof of (1.1). 


\section{Acknowledgement}

We thank the referee for bringing references $[2,3]$ to our attention.

\section{REFERENCES}

1. Aiki, T. Behavior of free boundaries of blow-up solutions to one-phase Stefan problems. Nonlinear Anal. 26, (1996) 707-723.

2. AIKI, T. \& IMAI, H. Global existence of solutions to one-phase Stefan problems for semilinear parabolic equations. Ann. Mat. Pura Appl. (IV) 175, (1998) 327-337.

3. AIKI, T. \& IMAI, H. Stability of global solutions to one-phase Stefan problems for semilinear parabolic equations. Czech. Math. J. 50, (2000) 135-153.

4. FASANO, A. \& PRIMICERIO, M. Free boundary problems for nonlinear parabolic equations with nonlinear free boundary conditions. J. Math. Anal. Appl. 72, (1979) 247-273.

5. Ghidouche, H., Souplet, Ph., \& Tarzia, D. Decay of global solutions, stability and blow-up for a reaction-diffusion problem with free boundary. Proc. Am. Math. Soc. 129, (2001) 781-792.

6. GigA, Y. A bound for global solutions of semilinear heat equations. Commun. Math. Phys. 103, (1986) $415-421$.

7. IMAI, H. \& KAWARADA, H. Numerical analysis of a free boundary problem involving blow-up phenomena. Proc. Joint Symp. Appl. Math. (1987) 277-280.

8. Souplet, PH. Stability and continuous dependence of solutions to one-phase Stefan problems for semilinear parabolic equations. Portugaliae Math., to appear. 